\title{
A new application of the unified method
}

\author{
Samil Akcagil ${ }^{* 1}$ and Tugba Aydemir ${ }^{2}$ \\ ${ }^{1}$ Vocational School of Pazaryeri, Bilecik Şeyh Edebali University, Bilecik, Turkey \\ ${ }^{2}$ Rectorate, Yalova University, Yalova, Turkey
}

Received: 14 May 2017, Accepted: 26 February 2018

Published online: 13 March 2018.

\begin{abstract}
In this paper, we show that the unified method is not only more general than the family of tanh function methods, also it gives many more general solutions than the new members of the family of $\left(\frac{G^{\prime}}{G}\right)$-expansion methods. Compared to other methods, the significant contribution of the unified method is firstly to unify the family of tanh function methods and the family of $\left(\frac{G^{\prime}}{G}\right)$-expansion methods. Secondly, it gives many more solutions for NPDEs direct, concise and simple manner than the total of these two families. Also, the unified method gives these abundant solutions without using tedious and complex algorithm on computer programs. Afterwards, we demonstrate the effectiveness of the unified tanh method by seeking more exact solutions of the Lonngren wave equation.
\end{abstract}

Keywords: The tanh function method, $\left(\frac{G^{\prime}}{G}\right)$-expansion method, the new approach of generalized $\left(\frac{G^{\prime}}{G}\right)$-expansion method, The $\left(\frac{G^{\prime}}{G}, \frac{1}{G}\right)$-expansion method, nonlinear partial differential equations, travelling wave solution, the unified method, the Lonngren wave equation.

\section{Introduction}

Nonlinear partial differential equations (NPDEs) have been subjected of study in various branches of mathematical physical sciences such as physics, biology, chemistry. Therefore, finding new methods and obtaining many more solutions for NPDEs plays an important role in science.

In recent years, tanh method and $\left(\frac{G^{\prime}}{G}\right)$-expansion method have been used many authors. The former is firstly introduced by Malfliet [1] and used in some paper [2-14] deriving different variations. The latter has been firstly introduced by Wang and Zhang [15] and widely used in some papers [16 - 24]. Although compared and showed the merits and demerits of these two methods and theirs variations in some papers [25-28], it is not enough to use only one type of these methods to find more solutions so far. Namely, it can be needed to use more than one type method to have more solutions for NPDEs. Therefore, we have considered to find a unification for these methods.

It is claimed that $\left(\frac{G^{\prime}}{G}\right)$-expansion method introduced by Wang and Zhang [15] gives many solutions for NPDEs than the family of tanh function methods. However, some authors as we have mentioned above showed that the solutions of $\left(\frac{G^{\prime}}{G}\right)$-expansion method and the modified extended tanh function method are exactly same [25-28]. The concrete relation of these two methods was given in our article [29]. To convert the solutions of $\left(\frac{G^{\prime}}{G}\right)$-expansion method to the solutions of the modified extended tanh function and to indicate the relation of coefficients and auxillary equations, the main point is to use transformation and trigonometric and hyperbolic identities. In our another article, we unified the family of tanh function methods under one method called the unified method [30]. 
Our main contribution in this paper is to propose the unified method as a unification also for the family of $\left(\frac{G^{\prime}}{G}\right)$-expansion methods. Therefore, it has been noticed the unified method gives many more solution for NPDEs without making too much effort on computer programs.

This paper is organized as follows. In section 2, we have presented description of the unified method. In section 3, we have showed and proved that the unified method unifies the family of $\left(\frac{G^{\prime}}{G}\right)$-expansion methods and gives many more solutions than any members of the family of these methods. In section 4, we have implemented the unified method to solve the Lonngren wave equation to obtain new solutions which could not be attained before.

\section{The unified method}

We describe the unified method for finding travelling wave solutions of NPDEs in the following steps. Suppose that a nonlinear partial differential equation(NPDE), say in two independent variables $x$ and $t$, is given by

$$
P\left(u, u_{t}, u_{x}, u_{x t}, u_{t t}, u_{x x}, \ldots\right)=0
$$

where $u(x, t)$ is an unknown function, $P$ is a polynomial in $u=u(x, t)$ and its various partial derivatives, in which highest order derivative and nonlinear terms are involved.

The summary of the unified method can be presented in the following six steps:

Step 1: To find the travelling wave solutions of Eq.(1), using the wave variable

$$
u(x, t)=U(\xi), \xi=x-c t
$$

where the constant $c$ is generally termed the wave velocity. Substituting Eq. (2) into Eq. (1), it is obtained the following ordinary differential equation(ODE) in $\xi$ (which illustrates a principal advantage of a travelling wave solution, i.e., a PDE is reduced to an ODE).

$$
P\left(U, c U^{\prime}, U^{\prime}, c U^{\prime \prime}, c^{2} U^{\prime \prime}, U^{\prime \prime}, \ldots\right)=0
$$

Step 2: If necessary one integrates Eq.(3) as many times as possible and set the constants of integration to be zero for simplicity.

Step 3: Supposed the solution of nonlinear partial differential equation can be expressed by an ansatz as follows:

$$
u(\xi)=a_{0}+\sum_{i=1}^{M}\left[a_{i} \phi^{i}+b_{i} \phi^{-i}\right]
$$

where $\phi=\phi(\xi)$ satisfies the Riccati differential equation

$$
\phi^{\prime}(\xi)=\phi^{2}(\xi)+b
$$

where $\phi^{\prime}=\frac{d \phi}{d \xi}$, and $a_{i}, b_{i}$ and $b$ are constants. The general solution of Eq. (5) as follows: 
Family 1: When $b<0$, the solutions of Eq. (5)

$$
\phi(\xi)=\left\{\begin{array}{l}
\frac{\sqrt{-\left(A^{2}+B^{2}\right) b}-A \sqrt{-b} \cosh \left(2 \sqrt{-b}\left(\xi+\xi_{0}\right)\right)}{A \sinh \left(2 \sqrt{-b}\left(\xi+\xi_{0}\right)\right)+B} \\
\frac{-\sqrt{-\left(A^{2}+B^{2}\right) b}-A \sqrt{-b} \cosh \left(2 \sqrt{-b}\left(\xi+\xi_{0}\right)\right)}{A \sinh \left(2 \sqrt{-b}\left(\xi+\xi_{0}\right)\right)+B} \\
\sqrt{-b}+\frac{-2 A \sqrt{-b}}{A+\cosh \left(2 \sqrt{-b}\left(\xi+\xi_{0}\right)\right)-\sinh \left(2 \sqrt{-b}\left(\xi+\xi_{0}\right)\right)} \\
-\sqrt{-b}+\frac{2 A \sqrt{-b}}{A+\cosh \left(2 \sqrt{-b}\left(\xi+\xi_{0}\right)\right)+\sinh \left(2 \sqrt{-b}\left(\xi+\xi_{0}\right)\right)}
\end{array}\right.
$$

where $A$ and $B$ are two real arbitrary constants, and $\xi_{0}$ arbitrary constant.

Family 2: When $b>0$, the solutions of Eq. (5)

$$
\phi(\xi)=\left\{\begin{array}{l}
\frac{\sqrt{\left(A^{2}-B^{2}\right) b}-A \sqrt{b} \cos \left(2 \sqrt{b}\left(\xi+\xi_{0}\right)\right)}{A \sin \left(2 \sqrt{b}\left(\xi+\xi_{0}\right)\right)+B} \\
\frac{-\sqrt{\left(A^{2}-B^{2}\right) b}-A \sqrt{b} \cos \left(2 \sqrt{b}\left(\xi+\xi_{0}\right)\right)}{A \sin \left(2 \sqrt{b}\left(\xi+\xi_{0}\right)\right)+B} \\
i \sqrt{b}+\frac{-2 A i \sqrt{b}}{A+\cos \left(2 \sqrt{b}\left(\xi+\xi_{0}\right)\right)-i \sin \left(2 \sqrt{b}\left(\xi+\xi_{0}\right)\right)} \\
-i \sqrt{b}+\frac{2 A i \sqrt{b}}{A+\cos \left(2 \sqrt{b}\left(\xi+\xi_{0}\right)\right)+i \sin \left(2 \sqrt{b}\left(\xi+\xi_{0}\right)\right)}
\end{array}\right.
$$

where $A$ and $B$ are two real arbitrary constants, and $\xi_{0}$ arbitrary constant.

Family 3: When $b=0$, the solution of Eq. (5)

$$
\phi(\xi)=-\frac{1}{\xi+\xi_{0}}
$$

where $\xi_{0}$ arbitrary constant.

Family 4: The positive integer $M$ can be accomplished by considering the homogeneous balance between the linear term of the highest order with the nonlinear term of highest degree appearing in Eq. (3) as follows:

If it is defined the degree of $u(\xi)$ as $D[u(\xi)]=M$, then the degree of other expressions is defined by

$$
\begin{gathered}
D\left[\frac{d^{q} u}{d \xi^{q}}\right]=M+q, \\
D\left[u^{r}\left(\frac{d^{q} u}{d \xi^{q}}\right)^{s}\right]=M r+s(q+M) .
\end{gathered}
$$

Therefore, it is found the value of $M$ in Eq. (4).

Step 4: Substituting Eq.(4) and (5) into Eq.(3) and collecting all terms with the same degrees of $\phi$ together, then setting each coeffcients of terms with $\phi^{i}(-M \leq i \leq M)$ to zero yield a set of algebraic equations for $a_{i}, b_{i}, c$ and $b$.

Step 5: Substituting $a_{i}, b_{i}, c$ and $b$ obtained in step 5 into (4) and using the general solutions of Eq.(5) in (6),(7) and (8), it can be obtained the explicit solutions of Eq.(1) immediately depending on the value of $b$.

\section{Comparison of the methods}

In mathematical literature, the family of tanh function methods and the family of $\left(\frac{G^{\prime}}{G}\right)$-expansion methods have been used widely to solve NPDEs over 20 years. Both of these methods were modified and developed in time.

$\left(\frac{G^{\prime}}{G}\right)$-expansion method has been modified and developed such as tanh function method in time and given new solutions 
for NPDEs if compared to modified extended tanh function method. The new approach of generalized $\left(\frac{G^{\prime}}{G}\right)$-expansion $[31-36]$ and the $\left(\frac{G^{\prime}}{G}, \frac{1}{G}\right)$-expansion method $[37-44]$ are two of them.

We show that the unified method unifies the new approach of generalized $\left(\frac{G^{\prime}}{G}\right)$-expansion method and the $\left(\frac{G^{\prime}}{G}, \frac{1}{G}\right)$-expansion method and it also gives many more solutions than these methods have.

\subsection{Comparison between the new approach of generalized $\left(\frac{G^{\prime}}{G}\right)$-expansion method and the unified method}

Theorem 1. The unified method gives many more solutions than the new approach of generalized $\left(\frac{G^{\prime}}{G}\right)$-expansion method.

Proof. If we divide the auxillary equation of the new approach of generalized $\left(\frac{G^{\prime}}{G}\right)$-expansion method

$$
G^{\prime \prime}(\xi)=A G^{2}(\xi)+B G(\xi) G^{\prime}(\xi)+C\left(G^{\prime}(\xi)\right)^{2}
$$

by $G^{2}(\xi)$ and then use the simple equality

$$
\frac{G^{\prime \prime}(\xi)}{G(\xi)}=\left(\frac{G^{\prime}(\xi)}{G(\xi)}\right)^{\prime}+\left(\frac{G^{\prime}(\xi)}{G(\xi)}\right)^{2}
$$

we get

$$
\left(\frac{G^{\prime}(\xi)}{G(\xi)}\right)^{\prime}+\left(\frac{G^{\prime}(\xi)}{G(\xi)}\right)^{2}=A+B\left(\frac{G^{\prime}(\xi)}{G(\xi)}\right)+C\left(\frac{G^{\prime}(\xi)}{G(\xi)}\right)^{2} .
$$

The last equation can be written as follows:

$$
\left(\frac{G^{\prime}(\xi)}{G(\xi)}\right)^{\prime}=(C-1)\left(\frac{G^{\prime}(\xi)}{G(\xi)}+\frac{B}{2(C-1)}\right)^{2}+\frac{4 A(C-1)-B^{2}}{4(C-1)}
$$

As it can be seen that Eq. (10) is completely equal to equation

$$
\phi(\xi)^{\prime}=a \phi(\xi)^{2}+b
$$

when considered

$$
\phi(\xi)=\frac{G^{\prime}(\xi)}{G(\xi)}+\frac{B}{2(C-1)}, a=C-1, b=\frac{4 A(C-1)-B^{2}}{4(C-1)} .
$$

From here, it has been reached the connection between the Eq.s (10) and (11) that they are essentially the same equation. To compensate the difference in the solutions in (6) and (7), it can be put to the coefficients $\frac{\sqrt{\mp a b}}{a}$ instead of $\mp b$ outside of functions and $\sqrt{\mp a b}$ instead of $\mp b$ inside of functions. This operation converts all of the solutions of (5) to solutions of (10).

After taking the following hyperbolic and trigonometric identities into consideration and using the binomial expansion and auxiliary transformation

$$
\frac{G^{\prime}(\xi)}{G(\xi)}=\phi(\xi)+\frac{B}{2(1-C)}
$$


we can gain a general formula that gives a connection between the coefficients of these two methods. Considering the ansatz of the new approach of generalized $\left(\frac{G^{\prime}}{G}\right)$-expansion method

$$
u(\xi)=\sum_{i=0}^{M} a_{i}\left(\frac{G^{\prime}(\xi)}{G(\xi)}\right)^{i}
$$

and of the unified tanh method

$$
u(\xi)=A_{0}+\sum_{i=1}^{M}\left[A_{i} \phi^{i}(\xi)+B_{i} \phi^{-i}(\xi)\right]
$$

as follows:

$$
u(\xi)=\sum_{i=0}^{M} a_{i}\left(\frac{G^{\prime}(\xi)}{G(\xi)}\right)^{i}=\sum_{i=1}^{M} a_{i}\left(\phi(\xi)+\frac{B}{2(1-C)}\right)^{i}=A_{0}+\sum_{i=1}^{M}\left[A_{i} \phi^{i}(\xi)+B_{i} \phi^{-i}(\xi)\right]
$$

so that gives

$$
A_{n}=\sum_{i=n}^{M}\left[\left(\begin{array}{c}
i \\
i-n
\end{array}\right)\left(\frac{B}{2(1-C)}\right)^{i-n} a_{i}\right], B_{n}=0
$$

From here, the solutions obtained by the new approach of generalized $\left(\frac{G^{\prime}}{G}\right)$-expansion method can be converted easily to the some solutions of the unified method. However, the unified method gives many more solutions which can not be obtained by the new approach of generalized $\left(\frac{G^{\prime}}{G}\right)$-expansion method. Particularly, the solutions consist of the functiontype in (6) and (7).

\subsection{Comparison between the $\left(\frac{G^{\prime}}{G}, \frac{1}{G}\right)$-expansion method and the unified method}

Theorem 2. The unified method gives many more solutions than the $\left(\frac{G^{\prime}}{G}, \frac{1}{G}\right)$-expansion method.

Proof. The $\left(\frac{G^{\prime}}{G}, \frac{1}{G}\right)$-expansion method, which can be thought of as an extension of the $\left(\frac{G^{\prime}}{G}\right)$-expansion method, gives the solutions as follows:

$$
u(\xi)=\left\{\begin{array}{c}
\sum_{i=0}^{M} a_{i}\left(\frac{A_{1} \cosh (k \xi)+A_{2} \sinh (k \xi)}{A_{1} \sinh (k \xi)+A_{2} \cosh (k \xi)+C}\right)^{i}+\sum_{i=1}^{M} b_{i} \frac{\left(A_{1} \cosh (k \xi)+A_{2} \sinh (k \xi)\right)^{i-1}}{\left(A_{1} \sinh (k \xi)+A_{2} \cosh (k \xi)+C\right)^{i}} \\
\sum_{i=0}^{M} a_{i}\left(\frac{A_{1} \cos (k \xi)-A_{2} \sin (k \xi)}{A_{1} \sin (k \xi)+A_{2} \cos (k \xi)+C}\right)^{i}+\sum_{i=1}^{M} b_{i} \frac{\left(A_{1} \sin (k \xi)-A_{2} \cos (k \xi)\right)^{i-1}}{\left(A_{1} \sin (k \xi)+A_{2} \cos (k \xi)+C\right)^{i}}
\end{array}\right\} .
$$

Using the hyperbolic and trigonometric identities

$$
\left\{\begin{array}{c}
\cosh (x \mp y)=\cosh (x) \cosh (y) \mp \sinh (x) \sinh (y) \\
\sinh (x \mp y)=\sinh (x) \cosh (y) \mp \cosh (x) \sinh (y) \\
\cos (x \mp y)=\cos (x) \cos (y) \pm \sin (x) \sin (y) \\
\sin (x \mp y)=\sin (x) \cos (y) \mp \cos (x) \sin (y)
\end{array}\right.
$$

and taking $A_{1}=\cosh \left(\xi_{0}\right), A_{2}=\sinh \left(\xi_{0}\right)$ such that $\tanh \left(\xi_{0}\right)=\frac{A_{2}}{A_{1}}$ and $A_{1}=\cos \left(\xi_{0}\right), A_{2}=\sin \left(\xi_{0}\right)$ such that $\tan \left(\xi_{0}\right)=\frac{A_{2}}{A_{1}}$, respectively in (14), the solutions in (14) can be converted easily the solutions the first two of (6) and (7) as follows:

$$
u(\xi)=\left\{\begin{array}{c}
\sum_{i=0}^{M} a_{i}\left(\frac{\cosh \left(k\left(\xi+\xi_{0}\right)\right)}{\sinh \left(k\left(\xi+\xi_{0}\right)+C\right.}\right)^{i}+\sum_{i=1}^{M} b_{i} \frac{\left(\cosh \left(k\left(\xi+\xi_{0}\right)\right)^{i-1}\right.}{\left(\sinh \left(k\left(\xi+\xi_{0}\right)+C\right)^{i}\right.}=a_{0}+\sum_{i=1}^{M}\left(\frac{a_{i} \cosh ^{i}\left(k\left(\xi+\xi_{0}\right)\right)+b_{i} \cosh ^{i-1}\left(k\left(\xi+\xi_{0}\right)\right)}{\left(\sinh \left(k\left(\xi+\xi_{0}\right)\right)+C\right)^{i}}\right) \\
\sum_{i=0}^{M} a_{i}\left(\frac{\cos \left(k\left(\xi+\xi_{0}\right)\right)}{\sin \left(k\left(\xi+\xi_{0}\right)+C\right.}\right)^{i}+\sum_{i=1}^{M} b_{i} \frac{\left(\cos \left(k\left(\xi+\xi_{0}\right)\right)^{i-1}\right.}{\left(\sin \left(k\left(\xi+\xi_{0}\right)+C\right)^{i}\right.}=a_{0}+\sum_{i=1}^{M}\left(\frac{a_{i} \cos ^{i}\left(k\left(\xi+\xi_{0}\right)\right)+b_{i} \cos ^{i-1}\left(k\left(\xi+\xi_{0}\right)\right)}{\left(\sin \left(k\left(\xi+\xi_{0}\right)\right)+C\right)^{i}}\right)
\end{array}\right.
$$


Considering the solution types the first two in (6) and (7) with (4), the solutions of the unified method

$$
u(\xi)=\left\{\begin{array}{c}
A_{0}+\sum_{i=1}^{M}\left(A_{i}\left(-\frac{A \sqrt{-b} \cosh \left(2 \sqrt{-b}\left(\xi+\xi_{0}\right)\right) \pm \sqrt{-\left(A^{2}+B^{2}\right) b}}{A \sinh \left(2 \sqrt{-b}\left(\xi+\xi_{0}\right)\right)+B}\right)^{i}+B_{i}\left(-\frac{A \sqrt{-b} \cosh \left(2 \sqrt{-b}\left(\xi+\xi_{0}\right)\right) \pm \sqrt{-\left(A^{2}+B^{2}\right) b}}{A \sinh \left(2 \sqrt{-b}\left(\xi+\xi_{0}\right)\right)+B}\right)^{-i}\right) \\
A_{0}+\sum_{i=1}^{M}\left(A_{i}\left(-\frac{A \sqrt{b} \cos \left(2 \sqrt{b}\left(\xi+\xi_{0}\right)\right) \pm \sqrt{\left(A^{2}-B^{2}\right) b}}{A \sin \left(2 \sqrt{b}\left(\xi+\xi_{0}\right)\right)+B}\right)^{i}+B_{i}\left(-\frac{A \sqrt{b} \cos \left(2 \sqrt{b}\left(\xi+\xi_{0}\right)\right) \pm \sqrt{\left(A^{2}-B^{2}\right) b}}{A \sin \left(2 \sqrt{b}\left(\xi+\xi_{0}\right)\right)+B}\right)^{-i}\right)
\end{array}\right.
$$

are obtained. It can be noticed that (16) is the first two binomial expansion terms of the numerator of the first term in (17).

Therefore, we have proved that the $\left(\frac{G^{\prime}}{G}, \frac{1}{G}\right)$-expansion method does not give different solutions. On the contrary, it gives limited solutions with too much effort. However, the unified method, as mentioned previous section, gives many more solutions without needing unnecessary effort as other methods including the $\left(\frac{G^{\prime}}{G}, \frac{1}{G}\right)$-expansion method.

\section{The Lonngren wave equation as an illustrative example}

The Lonngren wave equation is given by

$$
\frac{\partial^{2}}{\partial t^{2}}\left(u_{x x}-\alpha u+\beta u^{2}\right)+u_{x x}=0 .
$$

which describes electric signals in telegraph lines on the basis of the tunnel diode [45-47]. Note that in the book [48], sufficient conditions of the blow-up for the corresponding initial-boundary-value problems were obtained.

Using the wave variable $\xi=x-c t$ in Eq.(18), then integrating this equation and considering the integration constant to be zero, we obtain

$$
c^{2} U^{\prime \prime}+\left(1-\alpha c^{2}\right) U+\beta c^{2} U^{2}=0
$$

Balancing $U^{2}$ and $U^{\prime \prime}$ gives $M=2$.

The solutions of Eq.(19) can be written in the form as in (7)

$$
U(\xi)=a_{0}+\sum_{i=1}^{2}\left[a_{i} \phi^{i}+b_{i} \phi^{-i}\right]
$$

where $a_{0}, a_{1}, a_{2}, b_{1}$ and $b_{2}$ are constants which are unknowns to be determined later.

Substituting Eq.(20) and its derivatives into Eq.(19) and equating each coefficient of $\phi$ to zero, we obtain a set of nonlinear algebraic equations for $a_{0}, a_{1}, a_{2}, b_{1}, b_{2}$ and $c$. Solving this system using Maple, we obtain

Set 1:

$$
c=\mp \frac{1}{\sqrt{\alpha+4 b}}, b_{2}=0=b_{1}, a_{2}=-\frac{6}{\beta}, a_{1}=0, a_{0}=-\frac{6 b}{\beta}
$$

Set 2:

$$
c=\mp \frac{1}{\sqrt{\alpha-4 b}}, b_{2}=0=b_{1}, a_{2}=-\frac{6}{\beta}, a_{1}=0, a_{0}=-\frac{2 b}{\beta}
$$

Set 3:

$$
c=\mp \frac{1}{\sqrt{\alpha-4 b}}, b_{2}=-\frac{6 b^{2}}{\beta}, b_{1}=0, a_{2}=0=a_{1}, a_{0}=-\frac{2 b}{\beta}
$$


Set 4:

$$
c=\mp \frac{1}{\sqrt{\alpha+4 b}}, b_{2}=-\frac{6 b^{2}}{\beta}, b_{1}=0, a_{2}=0=a_{1}, a_{0}=-\frac{6 b}{\beta} \text {; }
$$

Set 5:

$$
c=\mp \frac{1}{\sqrt{\alpha-16 b}}, b_{2}=-\frac{6 b^{2}}{\beta}, b_{1}=0, a_{2}=-\frac{6}{\beta}, a_{1}=0, a_{0}=\frac{4 b}{\beta} ;
$$

Set 6:

$$
c=\mp \frac{1}{\sqrt{\alpha+16 b}}, b_{2}=-\frac{6 b^{2}}{\beta}, b_{1}=0, a_{2}=-\frac{6}{\beta}, a_{1}=0, a_{0}=-\frac{12 b}{\beta} ;
$$

Using these values, we obtain following general solutions, respectively:

$$
\begin{aligned}
& u_{1}(x, t)=-\frac{6 b}{\beta}-\frac{6}{\beta}\left(\frac{\sqrt{-\left(A^{2}+B^{2}\right) b}-A \sqrt{-b} \cosh \left(2 \sqrt{-b}\left(x \mp \frac{t}{\sqrt{\alpha+4 b}}+\xi_{0}\right)\right)}{A \sinh \left(2 \sqrt{-b}\left(x \mp \frac{t}{\sqrt{\alpha+4 b}}+\xi_{0}\right)\right)+B}\right)^{2}, \\
& u_{2}(x, t)=-\frac{6 b}{\beta}-\frac{6}{\beta}\left(\frac{\sqrt{-\left(A^{2}+B^{2}\right) b}+A \sqrt{-b} \cosh \left(2 \sqrt{-b}\left(x \mp \frac{t}{\sqrt{\alpha+4 b}}+\xi_{0}\right)\right)}{A \sinh \left(2 \sqrt{-b}\left(x \mp \frac{t}{\sqrt{\alpha+4 b}}+\xi_{0}\right)\right)+B}\right)^{2}, \\
& u_{3}(x, t)=-\frac{6 b}{\beta}-\frac{6}{\beta}\left(\sqrt{-b}+\frac{-2 A \sqrt{-b}}{A+\cosh \left(2 \sqrt{-b}\left(x \mp \frac{t}{\sqrt{\alpha+4 b}}+\xi_{0}\right)\right)-\sinh \left(2 \sqrt{-b}\left(x \mp \frac{t}{\sqrt{\alpha+4 b}}+\xi_{0}\right)\right)}\right)^{2}, \\
& u_{4}(x, t)=-\frac{6 b}{\beta}-\frac{6}{\beta}\left(-\sqrt{-b}+\frac{2 A \sqrt{-b}}{A+\cosh \left(2 \sqrt{-b}\left(x \mp \frac{t}{\sqrt{\alpha+4 b}}+\xi_{0}\right)\right)+\sinh \left(2 \sqrt{-b}\left(x \mp \frac{t}{\sqrt{\alpha+4 b}}+\xi_{0}\right)\right)}\right)^{2}, \\
& u_{5}(x, t)=-\frac{2 b}{\beta}-\frac{6}{\beta}\left(\frac{\sqrt{-\left(A^{2}+B^{2}\right) b}-A \sqrt{-b} \cosh \left(2 \sqrt{-b}\left(x \mp \frac{t}{\sqrt{\alpha-4 b}}+\xi_{0}\right)\right)}{A \sinh \left(2 \sqrt{-b}\left(x \mp \frac{t}{\sqrt{\alpha-4 b}}+\xi_{0}\right)\right)+B}\right)^{2}, \\
& u_{6}(x, t)=-\frac{2 b}{\beta}-\frac{6}{\beta}\left(\frac{\sqrt{-\left(A^{2}+B^{2}\right) b}+A \sqrt{-b} \cosh \left(2 \sqrt{-b}\left(x \mp \frac{t}{\sqrt{\alpha-4 b}}+\xi_{0}\right)\right)}{A \sinh \left(2 \sqrt{-b}\left(x \mp \frac{t}{\sqrt{\alpha-4 b}}+\xi_{0}\right)\right)+B}\right)^{2}, \\
& u_{7}(x, t)=-\frac{2 b}{\beta}-\frac{6}{\beta}\left(\sqrt{-b}+\frac{-2 A \sqrt{-b}}{A+\cosh \left(2 \sqrt{-b}\left(x \mp \frac{t}{\sqrt{\alpha-4 b}}+\xi_{0}\right)\right)-\sinh \left(2 \sqrt{-b}\left(x \mp \frac{t}{\sqrt{\alpha-4 b}}+\xi_{0}\right)\right)}\right)^{2}, \\
& u_{8}(x, t)=-\frac{2 b}{\beta}-\frac{6}{\beta}\left(-\sqrt{-b}+\frac{2 A \sqrt{-b}}{A+\cosh \left(2 \sqrt{-b}\left(x \mp \frac{t}{\sqrt{\alpha+4 b}}+\xi_{0}\right)\right)+\sinh \left(2 \sqrt{-b}\left(x \mp \frac{t}{\sqrt{\alpha+4 b}}+\xi_{0}\right)\right)}\right)^{2} \\
& u_{9}(x, t)=-\frac{2 b}{\beta}-\frac{6 b^{2}}{\beta}\left(\frac{A \sinh \left(2 \sqrt{-b}\left(x \mp \frac{t}{\sqrt{\alpha-4 b}}+\xi_{0}\right)\right)+B}{\sqrt{-\left(A^{2}+B^{2}\right) b}-A \sqrt{-b} \cosh \left(2 \sqrt{-b}\left(x \mp \frac{t}{\sqrt{\alpha-4 b}}+\xi_{0}\right)\right)}\right)^{2} \\
& u_{10}(x, t)=-\frac{2 b}{\beta}-\frac{6 b^{2}}{\beta}\left(\frac{A \sinh \left(2 \sqrt{-b}\left(x \mp \frac{t}{\sqrt{\alpha-4 b}}+\xi_{0}\right)\right)+B}{\sqrt{-\left(A^{2}+B^{2}\right) b}+A \sqrt{-b} \cosh \left(2 \sqrt{-b}\left(x \mp \frac{t}{\sqrt{\alpha-4 b}}+\xi_{0}\right)\right)}\right)^{2}
\end{aligned}
$$




$$
\begin{aligned}
& u_{11}(x, t)=-\frac{2 b}{\beta}-\frac{6 b^{2}}{\beta} \frac{1}{\left(\sqrt{-b}+\frac{-2 A \sqrt{-b}}{A+\cosh \left(2 \sqrt{-b}\left(x \mp \frac{t}{\sqrt{\alpha-4 b}}+\xi_{0}\right)\right)-\sinh \left(2 \sqrt{-b}\left(x \mp \frac{t}{\sqrt{\alpha-4 b}}+\xi_{0}\right)\right)}\right)^{2}}, \\
& u_{12}(x, t)=-\frac{2 b}{\beta}-\frac{6 b^{2}}{\beta} \frac{1}{\left(-\sqrt{-b}+\frac{2 A \sqrt{-b}}{A+\cosh \left(2 \sqrt{-b}\left(x \mp \frac{t}{\sqrt{\alpha+4 b}}+\xi_{0}\right)\right)+\sinh \left(2 \sqrt{-b}\left(x \mp \frac{t}{\sqrt{\alpha+4 b}}+\xi_{0}\right)\right)}\right)^{2}} \text {, } \\
& u_{13}(x, t)=-\frac{6 b}{\beta}-\frac{6 b^{2}}{\beta}\left(\frac{A \sinh \left(2 \sqrt{-b}\left(x \mp \frac{t}{\sqrt{\alpha+4 b}}+\xi_{0}\right)\right)+B}{\sqrt{-\left(A^{2}+B^{2}\right) b}-A \sqrt{-b} \cosh \left(2 \sqrt{-b}\left(x \mp \frac{t}{\sqrt{\alpha+4 b}}+\xi_{0}\right)\right)}\right)^{2} \\
& u_{14}(x, t)=-\frac{6 b}{\beta}-\frac{6 b^{2}}{\beta}\left(\frac{A \sinh \left(2 \sqrt{-b}\left(x \mp \frac{t}{\sqrt{\alpha+4 b}}+\xi_{0}\right)\right)+B}{\sqrt{-\left(A^{2}+B^{2}\right) b}+A \sqrt{-b} \cosh \left(2 \sqrt{-b}\left(x \mp \frac{t}{\sqrt{\alpha+4 b}}+\xi_{0}\right)\right)}\right)^{2}
\end{aligned}
$$

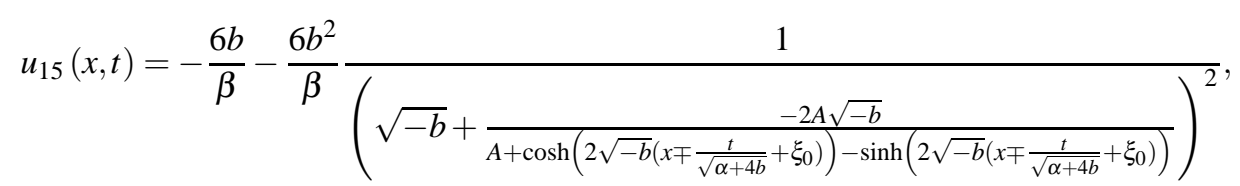

$$
\begin{aligned}
& \left.u_{16}(x, t)=-\frac{6 b}{\beta}-\frac{6 b^{2}}{\beta} \frac{1}{\left(-\sqrt{-b}+\frac{2 A \sqrt{-b}}{A+\cosh \left(2 \sqrt{-b}\left(x \mp \frac{t}{\sqrt{\alpha+4 b}}+\xi_{0}\right)\right)+\sinh \left(2 \sqrt{-b}\left(x \mp \frac{t}{\sqrt{\alpha+4 b}}+\xi_{0}\right)\right.}\right)}\right)^{2}, \\
& u_{17}(x, t)=\frac{4 b}{\beta}-\frac{6}{\beta}\left(\frac{\sqrt{-\left(A^{2}+B^{2}\right) b}-A \sqrt{-b} \cosh \left(2 \sqrt{-b}\left(x \mp \frac{t}{\sqrt{\alpha-16 b}}+\xi_{0}\right)\right)}{A \sinh \left(2 \sqrt{-b}\left(x \mp \frac{t}{\sqrt{\alpha-16 b}}+\xi_{0}\right)\right)+B}\right)^{2} \\
& -\frac{6 b^{2}}{\beta}\left(\frac{A \sinh \left(2 \sqrt{-b}\left(x \mp \frac{t}{\sqrt{\alpha-16 b}}+\xi_{0}\right)\right)+B}{\sqrt{-\left(A^{2}+B^{2}\right) b}-A \sqrt{-b} \cosh \left(2 \sqrt{-b}\left(x \mp \frac{t}{\sqrt{\alpha-16 b}}+\xi_{0}\right)\right)}\right)^{2}, \\
& u_{18}(x, t)=\frac{4 b}{\beta}-\frac{6}{\beta}\left(\frac{\sqrt{-\left(A^{2}+B^{2}\right) b}+A \sqrt{-b} \cosh \left(2 \sqrt{-b}\left(x \mp \frac{t}{\sqrt{\alpha-16 b}}+\xi_{0}\right)\right)}{A \sinh \left(2 \sqrt{-b}\left(x \mp \frac{t}{\sqrt{\alpha-16 b}}+\xi_{0}\right)\right)+B}\right)^{2} \\
& -\frac{6 b^{2}}{\beta}\left(\frac{A \sinh \left(2 \sqrt{-b}\left(x \mp \frac{t}{\sqrt{\alpha-16 b}}+\xi_{0}\right)\right)+B}{\sqrt{-\left(A^{2}+B^{2}\right) b}+A \sqrt{-b} \cosh \left(2 \sqrt{-b}\left(x \mp \frac{t}{\sqrt{\alpha-16 b}}+\xi_{0}\right)\right)}\right)^{2}, \\
& u_{19}(x, t)=\frac{4 b}{\beta}-\frac{6}{\beta}\left(\sqrt{-b}+\frac{-2 A \sqrt{-b}}{A+\cosh \left(2 \sqrt{-b}\left(x \mp \frac{t}{\sqrt{\alpha-16 b}}+\xi_{0}\right)\right)-\sinh \left(2 \sqrt{-b}\left(x \mp \frac{t}{\sqrt{\alpha-16 b}}+\xi_{0}\right)\right)}\right)^{2} \\
& -\frac{6 b^{2}}{\beta} \frac{1}{\left(\sqrt{-b}+\frac{-2 A \sqrt{-b}}{A+\cosh \left(2 \sqrt{-b}\left(x \mp \frac{t}{\sqrt{\alpha-16 b}}+\xi_{0}\right)\right)-\sinh \left(2 \sqrt{-b}\left(x \mp \frac{t}{\sqrt{\alpha-16 b}}+\xi_{0}\right)\right)}\right)^{2}}
\end{aligned}
$$




$$
\begin{aligned}
& u_{20}(x, t)=\frac{4 b}{\beta}-\frac{6}{\beta}\left(-\sqrt{-b}+\frac{2 A \sqrt{-b}}{A+\cosh \left(2 \sqrt{-b}\left(x \mp \frac{t}{\sqrt{\alpha+4 b}}+\xi_{0}\right)\right)+\sinh \left(2 \sqrt{-b}\left(x \mp \frac{t}{\sqrt{\alpha+4 b}}+\xi_{0}\right)\right)}\right)^{2} \\
& -\frac{6 b^{2}}{\beta} \frac{1}{\left(-\sqrt{-b}+\frac{2 A \sqrt{-b}}{A+\cosh \left(2 \sqrt{-b}\left(x \mp \frac{t}{\sqrt{\alpha+4 b}}+\xi_{0}\right)\right)+\sinh \left(2 \sqrt{-b}\left(x \mp \frac{t}{\sqrt{\alpha+4 b}}+\xi_{0}\right)\right)}\right)^{2}} \\
& u_{21}(x, t)=-\frac{12 b}{\beta}-\frac{6}{\beta}\left(\frac{\sqrt{-\left(A^{2}+B^{2}\right) b}-A \sqrt{-b} \cosh \left(2 \sqrt{-b}\left(x \mp \frac{t}{\sqrt{\alpha+16 b}}+\xi_{0}\right)\right)}{A \sinh \left(2 \sqrt{-b}\left(x \mp \frac{t}{\sqrt{\alpha+16 b}}+\xi_{0}\right)\right)+B}\right)^{2} \\
& -\frac{6 b^{2}}{\beta}\left(\frac{A \sinh \left(2 \sqrt{-b}\left(x \mp \frac{t}{\sqrt{\alpha+16 b}}+\xi_{0}\right)\right)+B}{\sqrt{-\left(A^{2}+B^{2}\right) b}-A \sqrt{-b} \cosh \left(2 \sqrt{-b}\left(x \mp \frac{t}{\sqrt{\alpha+16 b}}+\xi_{0}\right)\right)}\right)^{2} \text {, } \\
& u_{22}(x, t)=-\frac{12 b}{\beta}-\frac{6}{\beta}\left(\frac{\sqrt{-\left(A^{2}+B^{2}\right) b}+A \sqrt{-b} \cosh \left(2 \sqrt{-b}\left(x \mp \frac{t}{\sqrt{\alpha+16 b}}+\xi_{0}\right)\right)}{A \sinh \left(2 \sqrt{-b}\left(x \mp \frac{t}{\sqrt{\alpha+16 b}}+\xi_{0}\right)\right)+B}\right)^{2} \\
& -\frac{6 b^{2}}{\beta}\left(\frac{A \sinh \left(2 \sqrt{-b}\left(x \mp \frac{t}{\sqrt{\alpha+16 b}}+\xi_{0}\right)\right)+B}{\sqrt{-\left(A^{2}+B^{2}\right) b}+A \sqrt{-b} \cosh \left(2 \sqrt{-b}\left(x \mp \frac{t}{\sqrt{\alpha+16 b}}+\xi_{0}\right)\right)}\right)^{2} \text {, } \\
& u_{23}(x, t)=-\frac{12 b}{\beta}-\frac{6}{\beta}\left(\sqrt{-b}+\frac{-2 A \sqrt{-b}}{A+\cosh \left(2 \sqrt{-b}\left(x \mp \frac{t}{\sqrt{\alpha+16 b}}+\xi_{0}\right)\right)-\sinh \left(2 \sqrt{-b}\left(x \mp \frac{t}{\sqrt{\alpha+16 b}}+\xi_{0}\right)\right)}\right)^{2} \\
& -\frac{6 b^{2}}{\beta} \frac{1}{\left(\sqrt{-b}+\frac{-2 A \sqrt{-b}}{A+\cosh \left(2 \sqrt{-b}\left(x \mp \frac{t}{\sqrt{\alpha+16 b}}+\xi_{0}\right)\right)-\sinh \left(2 \sqrt{-b}\left(x \mp \frac{t}{\sqrt{\alpha+16 b}}+\xi_{0}\right)\right)}\right)^{2}} \\
& u_{24}(x, t)=-\frac{12 b}{\beta}-\frac{6}{\beta}\left(-\sqrt{-b}+\frac{2 A \sqrt{-b}}{A+\cosh \left(2 \sqrt{-b}\left(x \mp \frac{t}{\sqrt{\alpha+4 b}}+\xi_{0}\right)\right)+\sinh \left(2 \sqrt{-b}\left(x \mp \frac{t}{\sqrt{\alpha+4 b}}+\xi_{0}\right)\right)}\right)^{2} \\
& -\frac{6 b^{2}}{\beta} \frac{1}{\left(-\sqrt{-b}+\frac{2 A \sqrt{-b}}{A+\cosh \left(2 \sqrt{-b}\left(x \mp \frac{t}{\sqrt{\alpha+4 b}}+\xi_{0}\right)\right)+\sinh \left(2 \sqrt{-b}\left(x \mp \frac{t}{\sqrt{\alpha+4 b}}+\xi_{0}\right)\right)}\right)^{2}}
\end{aligned}
$$

where $b<0$ and $A$ and $B$ are two real arbitrary constants;

$$
u_{25}(x, t)=-\frac{6 b}{\beta}-\frac{6}{\beta}\left(\frac{\sqrt{\left(A^{2}-B^{2}\right) b}-A \sqrt{b} \cos \left(2 \sqrt{b}\left(x \mp \frac{t}{\sqrt{\alpha+4 b}}+\xi_{0}\right)\right)}{A \sin \left(2 \sqrt{b}\left(x \mp \frac{t}{\sqrt{\alpha+4 b}}+\xi_{0}\right)\right)+B}\right)^{2},
$$




$$
\begin{aligned}
& u_{26}(x, t)=-\frac{6 b}{\beta}-\frac{6}{\beta}\left(\frac{\sqrt{\left(A^{2}-B^{2}\right) b}+A \sqrt{b} \cos \left(2 \sqrt{b}\left(x \mp \frac{t}{\sqrt{\alpha+4 b}}+\xi_{0}\right)\right)}{A \sin \left(2 \sqrt{b}\left(x \mp \frac{t}{\sqrt{\alpha+4 b}}+\xi_{0}\right)\right)+B}\right)^{2}, \\
& u_{27}(x, t)=-\frac{6 b}{\beta}-\frac{6}{\beta}\left(i \sqrt{b}+\frac{-2 A i \sqrt{b}}{A+\cos \left(2 \sqrt{b}\left(x \mp \frac{t}{\sqrt{\alpha+4 b}}+\xi_{0}\right)\right)-i \sin \left(2 \sqrt{b}\left(x \mp \frac{t}{\sqrt{\alpha+4 b}}+\xi_{0}\right)\right)}\right)^{2}, \\
& u_{28}(x, t)=-\frac{6 b}{\beta}-\frac{6}{\beta}\left(-i \sqrt{b}+\frac{2 A i \sqrt{b}}{A+\cos \left(2 \sqrt{b}\left(x \mp \frac{t}{\sqrt{\alpha+4 b}}+\xi_{0}\right)\right)+i \sin \left(2 \sqrt{b}\left(x \mp \frac{t}{\sqrt{\alpha+4 b}}+\xi_{0}\right)\right)}\right)^{2} \text {, } \\
& u_{29}(x, t)=-\frac{2 b}{\beta}-\frac{6}{\beta}\left(\frac{\sqrt{\left(A^{2}-B^{2}\right) b}+A \sqrt{b} \cos \left(2 \sqrt{b}\left(x \mp \frac{t}{\sqrt{\alpha-4 b}}+\xi_{0}\right)\right)}{A \sin \left(2 \sqrt{b}\left(x \mp \frac{t}{\sqrt{\alpha-4 b}}+\xi_{0}\right)\right)+B}\right)^{2}, \\
& u_{30}(x, t)=-\frac{2 b}{\beta}-\frac{6}{\beta}\left(\frac{\sqrt{\left(A^{2}-B^{2}\right) b}-A \sqrt{b} \cos \left(2 \sqrt{b}\left(x \mp \frac{t}{\sqrt{\alpha-4 b}}+\xi_{0}\right)\right)}{A \sin \left(2 \sqrt{b}\left(x \mp \frac{t}{\sqrt{\alpha-4 b}}+\xi_{0}\right)\right)+B}\right)^{2} \text {, } \\
& u_{31}(x, t)=-\frac{2 b}{\beta}-\frac{6}{\beta}\left(i \sqrt{b}+\frac{-2 A i \sqrt{b}}{A+\cos \left(2 \sqrt{b}\left(x \mp \frac{t}{\sqrt{\alpha-4 b}}+\xi_{0}\right)\right)-i \sin \left(2 \sqrt{b}\left(x \mp \frac{t}{\sqrt{\alpha-4 b}}+\xi_{0}\right)\right)}\right)^{2} \\
& u_{32}(x, t)=-\frac{2 b}{\beta}-\frac{6}{\beta}\left(-i \sqrt{b}+\frac{2 A i \sqrt{b}}{A+\cos \left(2 \sqrt{b}\left(x \mp \frac{t}{\sqrt{\alpha+4 b}}+\xi_{0}\right)\right)+i \sin \left(2 \sqrt{b}\left(x \mp \frac{t}{\sqrt{\alpha+4 b}}+\xi_{0}\right)\right)}\right)^{2} \text {, } \\
& u_{33}(x, t)=-\frac{2 b}{\beta}-\frac{6 b^{2}}{\beta}\left(\frac{A \sin \left(2 \sqrt{b}\left(x \mp \frac{t}{\sqrt{\alpha-4 b}}+\xi_{0}\right)\right)+B}{\sqrt{\left(A^{2}-B^{2}\right) b}-A \sqrt{b} \cos \left(2 \sqrt{b}\left(x \mp \frac{t}{\sqrt{\alpha-4 b}}+\xi_{0}\right)\right)}\right)^{2}, \\
& u_{34}(x, t)=-\frac{2 b}{\beta}-\frac{6 b^{2}}{\beta}\left(\frac{A \sin \left(2 \sqrt{b}\left(x \mp \frac{t}{\sqrt{\alpha-4 b}}+\xi_{0}\right)\right)+B}{\sqrt{\left(A^{2}-B^{2}\right) b}+A \sqrt{b} \cos \left(2 \sqrt{b}\left(x \mp \frac{t}{\sqrt{\alpha-4 b}}+\xi_{0}\right)\right)}\right)^{2} \text {, } \\
& u_{35}(x, t)=-\frac{2 b}{\beta}-\frac{6 b^{2}}{\beta\left(i \sqrt{b}+\frac{-2 A i \sqrt{b}}{A+\cos \left(2 \sqrt{b}\left(x \mp \frac{t}{\sqrt{\alpha-4 b}}+\xi_{0}\right)\right)-i \sin \left(2 \sqrt{b}\left(x \mp \frac{t}{\sqrt{\alpha-4 b}}+\xi_{0}\right)\right)}\right)^{2}}, \\
& u_{36}(x, t)=-\frac{2 b}{\beta}-\frac{6 b^{2}}{\beta\left(-i \sqrt{b}+\frac{2 A i \sqrt{b}}{A+\cos \left(2 \sqrt{b}\left(x \mp \frac{t}{\sqrt{\alpha+4 b}}+\xi_{0}\right)\right)+i \sin \left(2 \sqrt{b}\left(x \mp \frac{t}{\sqrt{\alpha+4 b}}+\xi_{0}\right)\right)}\right)^{2}}, \\
& u_{37}(x, t)=-\frac{6 b}{\beta}-\frac{6 b^{2}}{\beta}\left(\frac{A \sin \left(2 \sqrt{b}\left(x \mp \frac{t}{\sqrt{\alpha+4 b}}+\xi_{0}\right)\right)+B}{\sqrt{\left(A^{2}-B^{2}\right) b}-A \sqrt{b} \cos \left(2 \sqrt{b}\left(x \mp \frac{t}{\sqrt{\alpha+4 b}}+\xi_{0}\right)\right)}\right)^{2} \text {, } \\
& u_{38}(x, t)=-\frac{6 b}{\beta}-\frac{6 b^{2}}{\beta}\left(\frac{A \sin \left(2 \sqrt{b}\left(x \mp \frac{t}{\sqrt{\alpha+4 b}}+\xi_{0}\right)\right)+B}{\sqrt{\left(A^{2}-B^{2}\right) b}+A \sqrt{b} \cos \left(2 \sqrt{b}\left(x \mp \frac{t}{\sqrt{\alpha+4 b}}+\xi_{0}\right)\right)}\right)^{2} \text {, }
\end{aligned}
$$




$$
\begin{aligned}
& u_{39}(x, t)=-\frac{6 b}{\beta}-\frac{6 b^{2}}{\beta\left(i \sqrt{b}+\frac{-2 A i \sqrt{b}}{A+\cos \left(2 \sqrt{b}\left(x \mp \frac{t}{\sqrt{\alpha+4 b}}+\xi_{0}\right)\right)-i \sin \left(2 \sqrt{b}\left(x \mp \frac{t}{\sqrt{\alpha+4 b}}+\xi_{0}\right)\right)}\right)^{2}}, \\
& u_{40}(x, t)=-\frac{6 b}{\beta}-\frac{6 b^{2}}{\beta\left(-i \sqrt{b}+\frac{2 A i \sqrt{b}}{A+\cos \left(2 \sqrt{b}\left(x \mp \frac{t}{\sqrt{\alpha+4 b}}+\xi_{0}\right)\right)+i \sin \left(2 \sqrt{b}\left(x \mp \frac{t}{\sqrt{\alpha+4 b}}+\xi_{0}\right)\right)}\right)^{2}}, \\
& u_{41}(x, t)=\frac{4 b}{\beta}-\frac{6}{\beta}\left(\frac{\sqrt{\left(A^{2}-B^{2}\right) b}-A \sqrt{b} \cos \left(2 \sqrt{b}\left(x \mp \frac{t}{\sqrt{\alpha-16 b}}+\xi_{0}\right)\right)}{A \sin \left(2 \sqrt{b}\left(x \mp \frac{t}{\sqrt{\alpha-16 b}}+\xi_{0}\right)\right)+B}\right)^{2} \\
& -\frac{6 b^{2}}{\beta}\left(\frac{A \sin \left(2 \sqrt{b}\left(x \mp \frac{t}{\sqrt{\alpha-16 b}}+\xi_{0}\right)\right)+B}{\sqrt{\left(A^{2}-B^{2}\right) b}-A \sqrt{b} \cos \left(2 \sqrt{b}\left(x \mp \frac{t}{\sqrt{\alpha-16 b}}+\xi_{0}\right)\right)}\right)^{2}, \\
& u_{42}(x, t)=\frac{4 b}{\beta}-\frac{6}{\beta}\left(\frac{\sqrt{\left(A^{2}-B^{2}\right) b}+A \sqrt{b} \cos \left(2 \sqrt{b}\left(x \mp \frac{t}{\sqrt{\alpha-16 b}}+\xi_{0}\right)\right)}{A \sin \left(2 \sqrt{b}\left(x \mp \frac{t}{\sqrt{\alpha-16 b}}+\xi_{0}\right)\right)+B}\right)^{2} \\
& -\frac{6 b^{2}}{\beta}\left(\frac{A \sin \left(2 \sqrt{b}\left(x \mp \frac{t}{\sqrt{\alpha-16 b}}+\xi_{0}\right)\right)+B}{\sqrt{\left(A^{2}-B^{2}\right) b}+A \sqrt{b} \cos \left(2 \sqrt{b}\left(x \mp \frac{t}{\sqrt{\alpha-16 b}}+\xi_{0}\right)\right)}\right)^{2}, \\
& u_{43}(x, t)=\frac{4 b}{\beta}-\frac{6}{\beta}\left(i \sqrt{b}+\frac{-2 A i \sqrt{b}}{A+\cos \left(2 \sqrt{b}\left(x \mp \frac{t}{\sqrt{\alpha-16 b}}+\xi_{0}\right)\right)-i \sin \left(2 \sqrt{b}\left(x \mp \frac{t}{\sqrt{\alpha-16 b}}+\xi_{0}\right)\right)}\right)^{2} \\
& -\frac{6 b^{2}}{\beta\left(i \sqrt{b}+\frac{-2 A i \sqrt{b}}{A+\cos \left(2 \sqrt{b}\left(x \mp \frac{t}{\sqrt{\alpha-16 b}}+\xi_{0}\right)\right)-i \sin \left(2 \sqrt{b}\left(x \mp \frac{t}{\sqrt{\alpha-16 b}}+\xi_{0}\right)\right)}\right)^{2}}, \\
& u_{44}(x, t)=\frac{4 b}{\beta}-\frac{6}{\beta}\left(-i \sqrt{b}+\frac{2 A i \sqrt{b}}{A+\cos \left(2 \sqrt{b}\left(x \mp \frac{t}{\sqrt{\alpha+4 b}}+\xi_{0}\right)\right)+i \sin \left(2 \sqrt{b}\left(x \mp \frac{t}{\sqrt{\alpha+4 b}}+\xi_{0}\right)\right)}\right)^{2} \\
& 6 b^{2}
\end{aligned}
$$

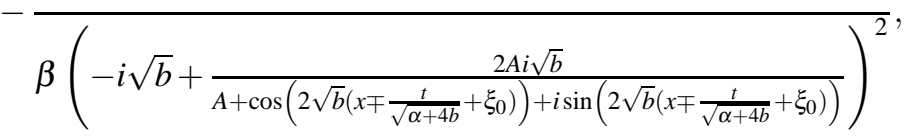




$$
\begin{aligned}
& u_{45}(x, t)=-\frac{12 b}{\beta}-\frac{6}{\beta}\left(\frac{\sqrt{\left(A^{2}-B^{2}\right) b}-A \sqrt{b} \cos \left(2 \sqrt{b}\left(x \mp \frac{t}{\sqrt{\alpha+16 b}}+\xi_{0}\right)\right)}{A \sin \left(2 \sqrt{b}\left(x \mp \frac{t}{\sqrt{\alpha+16 b}}+\xi_{0}\right)\right)+B}\right)^{2} \\
& -\frac{6 b^{2}}{\beta}\left(\frac{A \sin \left(2 \sqrt{b}\left(x \mp \frac{t}{\sqrt{\alpha+16 b}}+\xi_{0}\right)\right)+B}{\sqrt{\left(A^{2}-B^{2}\right) b}-A \sqrt{b} \cos \left(2 \sqrt{b}\left(x \mp \frac{t}{\sqrt{\alpha+16 b}}+\xi_{0}\right)\right)}\right)^{2}, \\
& u_{46}(x, t)=-\frac{12 b}{\beta}-\frac{6}{\beta}\left(\frac{\sqrt{\left(A^{2}-B^{2}\right) b}+A \sqrt{b} \cos \left(2 \sqrt{b}\left(x \mp \frac{t}{\sqrt{\alpha+16 b}}+\xi_{0}\right)\right)}{A \sin \left(2 \sqrt{b}\left(x \mp \frac{t}{\sqrt{\alpha+16 b}}+\xi_{0}\right)\right)+B}\right)^{2} \\
& -\frac{6 b^{2}}{\beta}\left(\frac{A \sin \left(2 \sqrt{b}\left(x \mp \frac{t}{\sqrt{\alpha+16 b}}+\xi_{0}\right)\right)+B}{\sqrt{\left(A^{2}-B^{2}\right) b}+A \sqrt{b} \cos \left(2 \sqrt{b}\left(x \mp \frac{t}{\sqrt{\alpha+16 b}}+\xi_{0}\right)\right)}\right)^{2}, \\
& u_{47}(x, t)=-\frac{12 b}{\beta}-\frac{6}{\beta}\left(i \sqrt{b}+\frac{-2 A i \sqrt{b}}{A+\cos \left(2 \sqrt{b}\left(x \mp \frac{t}{\sqrt{\alpha+16 b}}+\xi_{0}\right)\right)-i \sin \left(2 \sqrt{b}\left(x \mp \frac{t}{\sqrt{\alpha+16 b}}+\xi_{0}\right)\right)}\right)^{2} \\
& 6 b^{2}
\end{aligned}
$$

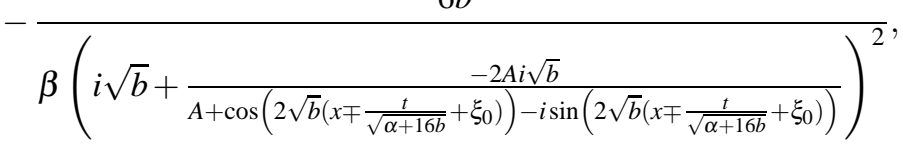

$$
\begin{aligned}
& u_{48}(x, t)=-\frac{12 b}{\beta}-\frac{6}{\beta}\left(-i \sqrt{b}+\frac{2 A i \sqrt{b}}{A+\cos \left(2 \sqrt{b}\left(x \mp \frac{t}{\sqrt{\alpha+4 b}}+\xi_{0}\right)\right)+i \sin \left(2 \sqrt{b}\left(x \mp \frac{t}{\sqrt{\alpha+4 b}}+\xi_{0}\right)\right)}\right)^{2} \\
& -\frac{6 b^{2}}{\beta\left(-i \sqrt{b}+\frac{2 A i \sqrt{b}}{A+\cos \left(2 \sqrt{b}\left(x \mp \frac{t}{\sqrt{\alpha+4 b}}+\xi_{0}\right)\right)+i \sin \left(2 \sqrt{b}\left(x \mp \frac{t}{\sqrt{\alpha+4 b}}+\xi_{0}\right)\right)}\right)^{2}},
\end{aligned}
$$

where $b>0$ and $A$ and $B$ are two real arbitrary constants;

$$
u_{49}(x, t)=-\frac{6}{\beta\left(x \mp \frac{t}{\sqrt{\alpha}}+\xi_{0}\right)^{2}},
$$

where $b=0$.

As it can be seen easily that unified method gives more than 49 solutions for the Lonngren wave equation when taking into account the positiveness or negativeness of values.

\section{Conclusions}

There are plenty of methods to solve nonlinear partial differential equations. The most used ones are the family of tanh function methods and the family of $\left(\frac{G^{\prime}}{G}\right)$-expansion methods. The main difficulty is if needed to have more solutions, it must have applied to some of them separately. Particulary, using some members of $\left(\frac{G^{\prime}}{G}\right)$-expansion method could be 
tedious and exhaustive algorithm at computer. In this study, using the unified method, we have aimed to unify and develop new and effective method to solve and have abundant solutions for NPDEs without being needed these two methods.

The advantages of the unified method, firstly it produces many more solutions than the other methods give. Namely, it gives not only the solutions of the other methods but also new exact solutions not obtained using other methods. Secondly, it unifies the merits of all the methods in one method without needing extra effort. Lastly, it has simple algorithm to apply on computer. Unlike the others, it reduces the process on computer as much as sometimes even calculated by hand.

To summarize briefly, the main and significant contributions of the method we construct in this paper are as follows:

(i) Using the unified method, it can be obtained many more solutions than total number of solutions of other methods by one method.

(ii) The unified method gives maximum different type solutions with minimum effort. On the other hand, it is claimed that "new solutions" have been found using some of the above methods; however, as mentioned in some articles [25 - 29], these "new solutions" are derived by extending some trigonometric or hyperbolic identities. Namely, they are disguised versions of the same solutions.

(iii) The unified method makes easier solving process at computer program. When using the unified method, it is not needed complex algorithm on computer programs. On the other hand, it is essential to use complex algorithm for some members of the $\left(\frac{G^{\prime}}{G}\right)$-expansion method.

The computations throughout this work were performed by using Maple.

\section{Competing interests}

The authors declare that they have no competing interests.

\section{Authors' contributions}

All authors have contributed to all parts of the article. All authors read and approved the final manuscript.

\section{References}

[1] W. Malfliet, Solitary wave solutions of nonlinear wave equations, Am. J. Phys. 60 (7) (1992) 650-654.

[2] W. Malfliet, W. Hereman, The tanh method. I: Exact solutions of nonlinear evolution and wave equations, Phys. Scr. 54 (1996) 563-568.

[3] W. Malfliet, W. Hereman, The tanh method. II: Perturbation technique for conservative systems, Phys. Scr. 54 (1996) $569-575$.

[4] W. Hereman, W. Malfliet, in: The tanh method: a tool to solve nonlinear partial differential equations with symbolic software, Proceedings 9th World Multi-Conference on Systemics, Cybernetics and Informatics, Orlando, FL, (2005), 165-168.

[5] A. M. Wazwaz, The tanh method for travelling wave solutions of nonlinear equations, Appl. Math. Comput. 154 (3) (2004) $713-$ 723.

[6] A. M. Wazwaz, The tanh method: exact solutions of the Sine-Gordon and the Sinh-Gordon equations, Appl. Math. Comput. 49 (2005) 565-574.

[7] A. M. Wazwaz, The tanh and the sine-cosine methods for a reliable treatment of the modified equal width equation and its variants, Comm. Nonlinear Sci. Numer. Simul. 11 (2) (2006) 148-160. 
[8] A. M. Wazwaz, The tanh and the sine-cosine methods for compact and noncompact solutions of the nonlinear Klein-Gordon equation, Appl. Math. Comput. 167 (2) (2005) 1179-1195.

[9] A. M. Wazwaz, The tanh method: solitons and periodic solutions for the Dodd-Bullough-Mikhailov and the Tzitzeica-DoddBullough equations, Chaos, Solitons Fractals 25 (1) (2005) 55-63.

[10] A. M. Wazwaz, The tanh method for generalized forms of nonlinear heat conduction and Burgers-Fisher equations, Appl. Math.Comput. 169 (2005) 321-338.

[11] A. M. Wazwaz, Travelling wave solutions of generalized forms of Burgers, Burgers-KdV and Burgers-Huxley equations, Appl. Math.Comput. 169 (2005) 639-656.

[12] A.M. Wazwaz, The extended tanh method for new solitons solutions for many forms of the fifth-order KdV equations, Appl. Math.Comput.,184, (2007), 1002-1014.

[13] A.M. Wazwaz, New solitary wave solutions to the modified forms of Degasperis-Procesi and Camass-Holm equations, Appl. Math.Comput., 186, (2007), 130-141.

[14] S.A. Khuri, A complex tanh-function method applied to nonlinear equations of Schrodinger type, Chaos, Solitons and Fractals 20 (2004), 1037-1040.

[15] M.Wang, X.Li, J.Zhang, The $\left(\frac{G^{\prime}}{G}\right)$ expansion method and travelling wave solutions of nonlinear evolution equations in mathematical physics, Physics Letter A 372 (2008), 417-423.

[16] A.Bekir, Application of the $\left(\frac{G^{\prime}}{G}\right)$ expansion method for nonlinear evolution equations, Physics Letter A 372 (2008) $3400-3406$.

[17] J.Zhang, X.Wei, A generalized $\left(\frac{G^{\prime}}{G}\right)$ expansion method and its applications, Physics Letter A 372 (2008), 3653-3658.

[18] I.Aslan, T.Öziş, Analytical study on two nonlinear evolution equations by using $\left(\frac{G^{\prime}}{G}\right)$ expansion method, Appl. Math. Comp. 209 (2009), 425-429.

[19] E.M.E.Zayed, The $\left(\frac{G^{\prime}}{G}\right)$ expansion method and its application to some nonlinear evolution equations, J. Appl. Math. Comp. 30 (2009), 89-103.

[20] E.M.E.Zayed, K.A.Gepreel, Some applications of the $\left(\frac{G^{\prime}}{G}\right)$ expansion method to nonlinear partial differential equations, Appl. Math. Comp. 212 (2009), 1-13.

[21] A.Borhanifar, A.M.Zamiri, Application of the $\left(\frac{G^{\prime}}{G}\right)$ expansion method for the Zhiber-Shabat equation and other related equations, Math. Comp. Model. 54 (2011), 2109-2116.

[22] Z. Ayati, Comparing between $\left(\frac{G^{\prime}}{G}\right)$ expansion method and tanh-method, Open Engineering, 4, (2014), 334-340.

[23] M. Shakeel, S. T. Mohyud-Din, Improved $\left(\frac{G^{\prime}}{G}\right)$-expansion and extended tanh methods for $(2+1)$-dimensional CalogeroBogoyavlenskii-Schiff equation, Alexandria Engineering Journal, 54 (2015), 27-33.

[24] E. M. E. Zayed, A further improved $\left(\frac{G^{\prime}}{G}\right)$-expansion method and the extended tanh-method for finding exact solutions of nonlinear PDEs, Journal WSEAS Transactions on Mathematics, 10 (2011), 56-64.

[25] N. A. Kudryashov, N.B. Loguinova, Be careful with the Exp-function method, Commun. Nonlinear Sci. Numer. Simulat. 14 (2009) 1881-1890.

[26] N. A. Kudryashov, On "new travelling wave solutions" of the KdV and KdV-Burgers equations, Commun. Nonlinear Sci. Numer. Simulat. 14 (2009) 1891-1900.

[27] N. A. Kudryashov, Seven common errors in finding exact solutions of nonlinear differential equations, Commun. Nonlinear Sci. Numer. Simulat. 14 (2009) 3507-3529.

[28] E. J. Parkes, Observations on the basic $\left(\frac{G^{\prime}}{G}\right)$ - expansion method method for finding solutions to nonlinear evolution equations, Applied Mathematics and Computation 217 (2010) 1759-1763.

[29] Ş. Akçağıl, T. Aydemir, Comparison between the $\left(\frac{G^{\prime}}{G}\right)$ - expansion method and the modified extended tanh method, Open Physics 14 (2016) 88-94.

[30] Ö.F. Gözükızıl, Ş. Akçă̆ıll, T. Aydemir, Unification of all hyperbolic tangent function methods, Open Physics 14 (2016) $524-541$.

[31] M. Shakeel, S.T. Mohyud-Din, New $\left(\frac{G^{\prime}}{G}\right)$-expansion method and its application to the Zakharov-Kuznetsov-Benjamin-BonaMahony(ZK-BBM) equation, Journal of the Association of Arab Universities for Basic and Applied Sciences 18 (2015) 66-81.

[32] Md.N. Alam, M.A. Akbar, The new approach of the generalized $\left(\frac{G^{\prime}}{G}\right)$-expansion method for nonlinear evolution equations, Ain Shams Engineering Journal 5(2) (2014) 595-603.

[33] Md.N. Alam, M.A. Akbar, S.T. Mohyud-Din, General traveling wave solutions of the strain wave equation in microstructured solids via the new approach of generalized $\left(\frac{G^{\prime}}{G}\right)$-expansion method, Alexandria Engineering Journal 53 (2014) 233-241. 
[34] H. Naher, F.A. Abdullah, New generalized and improved new approach of generalized $\left(\frac{G^{\prime}}{G}\right)$-expansion method for nonlinear evolution equations in mathematical physics, Journal of the Egyptian Mathematical Society 22(3) (2014) 390-395.

[35] Md.N. Alam, M.A. Akbar, Traveling wave solutions for the mKdV equation and the Gardner equations by new approach of the generalized $\left(\frac{G^{\prime}}{G}\right)$-expansion method, Journal of the Egyptian Mathematical Society 22(3) (2014) 402-406.

[36] X. Liu, W. Zhang, Z. Li, Application of improved $\left(\frac{G^{\prime}}{G}\right)$-expansion method to travelling wave solutions of two nonlinear evolution equations, Advances in Applied Mathematics and Mechanics 4(1),122-130.

[37] S. Demiray, Ö. Ünsal, A. Bekir, Exact solutions of nonlinear wave equations using $\left(\frac{G^{\prime}}{G}, \frac{1}{G}\right)$-expansion method, Journal of the Egyptian Mathematical Society 23(1) (2015) 78-84.

[38] M. Kaplan, A. Bekir, M.Özer, Solving nonlinear evolution equation system using two different methods, Open Physics 13 (2015) 383-388.

[39] L. L-xiao, L. E-qiang, W. M-liang, The $\left(\frac{G^{\prime}}{G}, \frac{1}{G}\right)$-expansion method and its application to travelling wave solutions of the Zakharov equations, Appl Math-A J Chinese Univ.25(4) (2010) 454-462.

[40] E.M.E. Zayed, S.A. Hoda Ibrahim, M.A.M. Abdelaziz, Traveling wave solutions of the nonlinear (3+1)-dimensional KadomtsevPetviashvili equation using the two variables $\left(\frac{G^{\prime}}{G}, \frac{1}{G}\right)$-expansion method, J Appl Math. 2012 (2012) 1-8.

[41] E.M.E Zayed, M.A.M Abdelaziz, The two-variable $\left(\frac{G^{\prime}}{G}, \frac{1}{G}\right)$-expansion method for solving the nonlinear KdV-mKdV equation, Math Probl Eng. 2012 (2012) 1-14.

[42] L. Ling-Xiao, W. Ming-Liang, The $\left(\frac{G^{\prime}}{G}, \frac{1}{G}\right)$-expansion method and travelling wave solutions for a higher-order nonlinear schrödinger equation, Appl Math Comput. 208(2) (2009) 440-445.

[43] J.I. Zhang, U.M. Wang, M.L. Wang, Exact solutions to the two nonlinear equations, Acta Physica Sinica 52(7) (2003) 1574-1578.

[44] I.E. Inan, Y. Ugurlu, M.Inc, New Applications of the ( $\left.\frac{G^{\prime}}{G}, \frac{1}{G}\right)$-Expansion Method, Acta Physica Polonica 128 (2015) $245-252$.

[45] M. I. Rabinovich, Izv. Vyssh. Uchebn. Zaved. Radiofizika 17 (1974), 477-510.

[46] M. I. Rabinovich and D. I. Trubetskov, Introduction in theory of waves, Nauka, Moscow, 1984.

[47] K. E. Lonngren, H. C. S. Hsuan, and W. F. Ames, On the soliton, invariant, and shock solutions of a fourth-order nonlinear equation, J. Math. Anal. Appl. 52 (1975), 538-545.

[48] M. O. Korpusov, Blow up in nonclassical nonlocal equations, URSS, Moscow, 2011. 